\title{
A Lagrangian framework for deriving triples and quadruples corrections to the CCSD energy
}

Janus J. Eriksen' , Kasper Kristensen, Thomas Kjærgaard, Poul Jørgensen, and Jürgen Gauss

Citation: The Journal of Chemical Physics 140, 064108 (2014); doi: 10.1063/1.4862501

View online: http://dx.doi.org/10.1063/1.4862501

View Table of Contents: http://aip.scitation.org/toc/jcp/140/6

Published by the American Institute of Physics

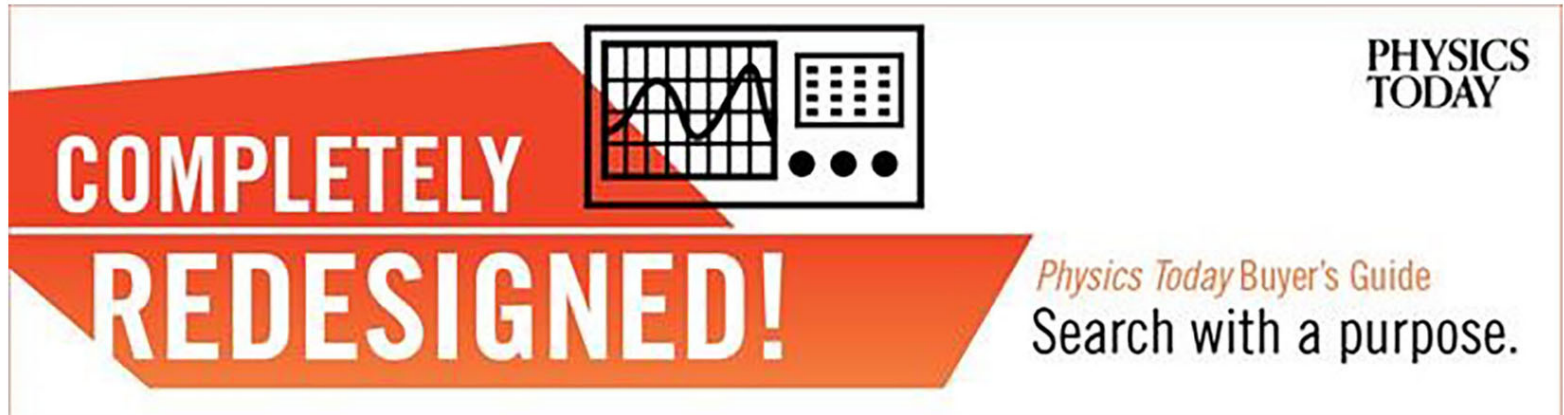




\title{
A Lagrangian framework for deriving triples and quadruples corrections to the CCSD energy
}

\author{
Janus J. Eriksen, ${ }^{1, a)}$ Kasper Kristensen, ${ }^{1}$ Thomas Kjærgaard, ${ }^{1}$ Poul Jørgensen, ${ }^{1}$ \\ and Jürgen Gauss ${ }^{2}$ \\ ${ }^{1}$ qLEAP Center for Theoretical Chemistry, Department of Chemistry, Aarhus University, Langelandsgade 140, \\ DK-8000 Aarhus C, Denmark \\ ${ }^{2}$ Institut für Physikalische Chemie, Johannes Gutenberg-Universität Mainz, Duesbergweg 10-14, \\ D-55128 Mainz, Germany
}

(Received 8 November 2013; accepted 6 January 2014; published online 12 February 2014)

\begin{abstract}
Using the coupled cluster Lagrangian technique, we have determined perturbative corrections to the coupled cluster singles and doubles (CCSD) energy that converge towards the coupled cluster singles, doubles, and triples (CCSDT) and coupled cluster singles, doubles, triples, and quadruples (CCSDTQ) energies, considering the CCSD state as the unperturbed reference state and the fluctuation potential as the perturbation. Since the Lagrangian technique is utilized, the energy corrections satisfy Wigner's $2 n+1$ rule for the cluster amplitudes and the $2 n+2$ rule for the Lagrange multipliers. The energy corrections define the CCSD perturbation series, CCSD $(\mathrm{T}-n)$ and CCSD(TQ $-n)$, which are term-wise size extensive to any order in the perturbation. A detailed comparison of the CCSD(TQ- $n$ ) series and the CC(2)PT( $n$ ) series of Hirata et al. [J. Chem. Phys. 114, 3919 (2001)] has been performed, revealing some deficiencies of the latter related to the target energy of the series and its lack of size extensivity. ( 2014 AIP Publishing LLC. [http://dx.doi.org/10.1063/1.4862501]
\end{abstract}

\section{INTRODUCTION}

The iterative coupled cluster ${ }^{1}$ (CC) singles and doubles ${ }^{2}$ (CCSD) model is a robust and successful ab initio electronic structure model that scales with system size, $N$, as $N^{6}$. In the CCSD model, the cluster operator is truncated at the level of double excitations. The inclusion of triple and quadruple excitations, however, is important for high accuracy electronic structure models, and significant efforts have therefore been invested in extending the CCSD model to include the effect of triple and quadruple ${ }^{3-23}$ excitations. The most popular approach has been to add the lowest-order triples energy contributions from many-body perturbation theory ${ }^{24}$ (MBPT) to the CCSD energy, resulting in non-iterative triples models that are correct through fourth order and contain some fifth-order candidate terms with respect to the Hartree-Fock state. These models aim at preserving the high accuracy of the $N^{8}$-scaling coupled cluster singles, doubles, and triples ${ }^{25}$ (CCSDT) model or the $N^{10}$-scaling coupled cluster singles, doubles, triples, and quadruples ${ }^{26}$ (CCSDTQ) model, in case of quadruples inclusion, without having to undertake any relaxation of triples or quadruples amplitudes, respectively, and accomplishing this using algorithms in which the scaling is governed by the most costly of the considered non-iterative MBPT contribution.

The most successful non-iterative triples model is the $\operatorname{CCSD}(\mathrm{T})$ model, which was introduced over 20 years $^{4}$ ago as an a posteriori correction to the CCSD energy. Preceding $\operatorname{CCSD}(T)$, alternative non-iterative triples models, e.g., the consistent fourth-order CCSD[T] model, ${ }^{3}$ had been tainted

\footnotetext{
a) Author to whom correspondence should be addressed. Electronic mail: janusje@chem.au.dk
}

from a consistent overestimation of triple excitation effects. This glaring inadequacy ${ }^{27}$ of perturbative triples models was alleviated with the introduction of the CCSD(T) model, which instantly acquired major recognition and has since become the "gold standard" of electronic structure theory. ${ }^{28}$ In the $\operatorname{CCSD}(\mathrm{T})$ model, the perturbative treatment of triple excitations is extended with respect to the CCSD[T] model by adding a (nearly always) positive fifth-order singles energy contribution, which delicately counterbalances ${ }^{5}$ the overestimation of the triples contribution entering in CCSD[T], often boasting an almost perfect error cancellation. Numerous attempts have been made at improving upon the $\operatorname{CCSD}(\mathrm{T})$ model by considering various fifth-order MBPT energy contributions, ${ }^{5-7}$ but the $\operatorname{CCSD}(\mathrm{T})$ model has hitherto remained the most successful of the approximate noniterative triples models. From a computational point of view, $\operatorname{CCSD}(\mathrm{T})$-alongside CCSD[T] — comes out superior to all alternative triples models as it only contains the minimum number of numerical steps that scale as $N^{7} .{ }^{4}$

Despite the success of the CCSD(T) model, many have challenged the lack of rigour in the derivation of the model and as years have gone by, more and more studies have featured deficient $\operatorname{CCSD}(\mathrm{T})$ results, e.g., for cases where the amount of static correlation involved cannot be neglected, see, for instance, Ref. 29. The conceptual scepticism surrounding the $\operatorname{CCSD}(\mathrm{T})$ model was alleviated with the emergence of the so-called asymmetric triples ${ }^{10-12}$ and quadruples ${ }^{11,30}$ models that allowed for the $\operatorname{CCSD}(\mathrm{T})$ model to be seen in a novel perspective. These asymmetric, non-iterative models respect and account for the non-Hermitian nature of the CC problem; namely, while both the ordinary Schrödinger equation and its adjoint form may be formulated as an eigenvalue problem, the resulting sets of right and left CC eigenstates are not 
identical. The asymmetric CCSD(T) model was simultaneously proposed by Kucharski and Bartlett ${ }^{10,11}$ as the $\Lambda \operatorname{CCSD}(\mathrm{T})$ model and by Crawford and $\operatorname{Stanton}^{12}$ as the a$\operatorname{CCSD}(\mathrm{T})$ model. The $\Lambda \mathrm{CCSD}(\mathrm{T})$ model is structurally similar to the ordinary $\operatorname{CCSD}(\mathrm{T})$ model, but expressed in terms of left state amplitudes rather than right state cluster amplitudes as in the $\operatorname{CCSD}(\mathrm{T})$ model. Recently, Taube and Bartlett ${ }^{29,31}$ have revitalized the $\Lambda \mathrm{CCSD}(\mathrm{T})$ model, comparing the performance of the model to that of the traditional CCSD(T) model as well as to that of other related triples models. Extensions of the above ideas to encompass corrections from quadruple excitations have likewise been proposed. The $\operatorname{CCSD}\left(\mathrm{TQ}_{f}\right)$ model of Kucharski and Bartlett ${ }^{11}$ has been proposed in an asymmetric form as $\Lambda \mathrm{CCSD}\left(\mathrm{TQ}_{f}\right),{ }^{30}$ and recently, generalizations of some of these approximations to higher excitations have been proposed by Kállay and Gauss. 22,23

The underlying philosophy behind the triples and quadruples corrections to the CCSD energy discussed so far have mostly been based on the selection of correction terms from the MBPT expansion, in which the Hartree-Fock (HF) state acts as the unperturbed reference state. However, if what we are interested in is to determine corrections directly to the CCSD energy, it is physically more sound to base these correction terms on the state in question, i.e., the CCSD state, as the unperturbed reference state. This observation has acted as a motivation for a plethora of studies, prominent examples of which have made use of the equation-of-motion coupled cluster (EOM-CC) engine ${ }^{32}$ as the framework for the derivation of non-iterative energy corrections.

The development of corrections derived from the EOMCC problem was initiated by Stanton ${ }^{33}$ who showed how the specific fifth-order MBPT singles term, which is added to the CCSD $[\mathrm{T}]$ correction in the CCSD(T) model, from an EOM$\mathrm{CC}$ perspective has to be taken into account among all of the MBPT fifth-order triples candidate contributions. In the analysis of Stanton, the CCSD state was considered as the zerothorder reference state and Löwdin's partitioning technique ${ }^{34}$ used to develop the triples corrections. In 2000, Gwaltney and Head-Gordon ${ }^{14}$ further developed upon the idea of deriving energy corrections using a Löwdin partitioning, resulting in a second-order correction to the CCSD energy known as the $\operatorname{CCSD}(2)$ model. Their derivation circumvented some of the assumptions that had been inherent to Ref. 33, and which were to some extent rooted in a traditional MBPT logic. The following year, the second-order energy correction was rederived by the same authors from the application of RayleighSchrödinger perturbation theory (RSPT) to the EOM-CC problem, ${ }^{15}$ a path which Hirata et al. ${ }^{16}$ also pursued in their related work on deriving a general perturbation $\mathrm{CC}(m) \mathrm{PT}(n)$ hierarchy. In the $\mathrm{CC}(m) \mathrm{PT}(n)$ hierarchy, the $\mathrm{CC}(2) \mathrm{PT}(2)$ (or $\left.\operatorname{CCSD}(2)_{\mathrm{TQ}}\right)$ model $^{17}$ is identical to the $\operatorname{CCSD}(2)$ model of Ref. 15 and includes, in addition to triples corrections, also corrections to the CCSD energy arising from quadruple excitations, while the $\mathrm{CC}(2) \mathrm{PT}(3)_{\mathrm{T}}$ and $\mathrm{CC}(2) \mathrm{PT}(3)_{\mathrm{TQ}}$ models (or $\operatorname{CCSD}(3)_{\mathrm{T}}$ and $\operatorname{CCSD}(3)_{\mathrm{TQ}}$, respectively) of Ref. 18 are third-order extensions.

A different, non-perturbative methodology has been proposed by Piecuch and co-workers, ${ }^{19-21}$ taking their stand also in the EOM-CC framework but focusing on developing lead- ing energy corrections by making approximations to the exact left state. This approach is denoted as a method of moments of CC equations (MMCC) and from it so-called renormalized and completely renormalized (CR) versions of, e.g., the $\operatorname{CCSD}(\mathrm{T})$ and $\operatorname{CCSD}(\mathrm{TQ})$ models have been derived. ${ }^{19}$ These CR-CCSD(T) and CR-CCSD(TQ) models are aimed at, and have in many cases been successful in, recovering some static correlation effects using only a single determinant reference state framework. The CR methods have been extended within a biorthogonal MMCC picture to a hierarchy of CR-CC $\left(m_{A}\right.$, $m_{B}$ ) models (e.g., the CR-CCSD $(\mathrm{T})_{\mathcal{L}}$ model) where the energy of a CC model truncated at excitation level $m_{A}$ is corrected from excitations of level $m_{B} \cdot{ }^{20,21}$

Finally, approximate non-iterative models that augment the full CCSDT energy have also been proposed, e.g., the CCSDT[Q] model of Kucharski and Bartlett ${ }^{7}$ and the CCSDT(Q) model of Bomble et al. ${ }^{13}$ where the former model was intuited from MBPT, while the latter was proposed following a strategy similar to the one Stanton ${ }^{33}$ employed to justify the $\operatorname{CCSD}(\mathrm{T})$ model. From the $\mathrm{CC}(m) \mathrm{PT}(n)$ hierarchy, the CC(3)PT(2) $\left(\operatorname{CCSDT}(2)_{Q}\right)^{17}$ model has been proposed, while the CR-CC $(3,4)$ is the leading-order correction to the CCSDT energy of the CR-CC $\left(m_{A}, m_{B}\right)$ hierarchy. ${ }^{20,21}$

In the present work, we derive triples and quadruples corrections to the CCSD energy by using as engine the technique of CC energy Lagrangians, in which cluster amplitudes and Lagrange multipliers constitute the variational parameters. By expanding the Lagrangian for the CCSDT or CCSDTQ energy around an expansion point represented by the CCSD right and left states, and performing a Møller-Plesset partitioning of the Hamiltonian, we derive corrections to the CCSD energy in orders of the fluctuation potential that cover effects from triple or triple and quadruple excitations, respectively. The resulting series, $\operatorname{CCSD}(\mathrm{T}-n)$ and CCSD(TQ- $n)$, converge towards the CCSDT or CCSDTQ limits, depending on what target energy is used in the derivation, and form two subseries of a general CC Lagrangian perturbation, $\mathrm{CC}\left[m_{1}\right]\left(\left[m_{2}\right]-n\right)$, hierarchy, where $\left[m_{1}\right]$ is the collection of excitation levels in the cluster operator of the parent CC model, $\left[m_{2}\right]$ the corresponding excitation levels of the target CC model, and $n$ the perturbation order.

The $\operatorname{CCSD}(\mathrm{T}-n)$ and $\operatorname{CCSD}(\mathrm{TQ}-n)$ perturbation series are compared to the $\mathrm{CC}(2) \mathrm{PT}(n)$ series, which is derived within the EOM-CC framework. The major difference between the present Lagrangian-based $\operatorname{CCSD}(\mathrm{T}-n)$ and CCSD(TQ- $n)$ series and the EOM-CC-based CC(2)PT $(n)$ series lies in the fact that whereas the right eigenstate of the Lagrangian-based series is parametrized in terms of an exponential cluster operator, it is in the series based upon EOM$\mathrm{CC}$ theory parametrized using linear cluster operators. The corrections to the parent CCSD energy of the Lagrangianbased series therefore converge towards a target CC energy, be that the CCSDT, CCSDTQ, etc., energy, depending on the energy referenced in the derivation of the series, while the series based on EOM-CC theory can only target the full configuration interaction $(\mathrm{FCI})$ energy, $E_{\mathrm{FCI}}$. Any pragmatic attempts at truncating the $\mathrm{CC}(2) \mathrm{PT}(n)$ series to a given excitation level, e.g., triples or quadruples, will inevitably violate the size extensivity of the EOM-CC problem due to issues 
similar to those encountered in truncated CI methods. ${ }^{35,36}$ Furthermore, such truncated series will fail to converge towards the CC energy corresponding to the truncation level. These problems are inherent to any EOM-CC perturbation series. Size extensivity problems, which are specific to the $\operatorname{CC}(2) \mathrm{PT}(n)$ series, occur due to the fact that the chosen zeroth-order Hamiltonian is not additively separable. As additive separability of the zeroth-order Hamiltonian is a requirement of any size-extensive perturbation series, this stands in contrast to the similarity-transformed Fock operator, which acts as the zeroth-order Hamiltonian in the Lagrangian-based series, and which is indeed additively separable. As a result, the energy corrections of the $\mathrm{CC}(2) \mathrm{PT}(n)$ series are in general not size extensive, regardless of whether the series is truncated or not. The differences between the $\mathrm{CC}(2) \mathrm{PT}(n)$ series - with a pragmatic truncation such that corrections from excitation levels higher than triples or quadruples are neglected-and the term-wise sizeextensive $\operatorname{CCSD}(\mathrm{T}-n) / \operatorname{CCSD}(\mathrm{TQ}-n)$ series begin at fourth order, owing not only to the different partitioning schemes used, but also to the different pictures in which they are derived.

In Sec. II, we prepare our investigation by briefly revisiting the concept of CC energy Lagrangians, followed by the application of this technique to the full CCSDTQ energy. After simplifications of the CCSDTQ energy Lagrangian from the right and left CCSD state equations, it is expanded in orders of the fluctuation potential and the CCSD(TQ- $n$ ) series, alongside a complementary $\operatorname{CCSD}(\mathrm{T}-n)$ series, is derived. Section III features comparisons between the present Lagrangian-based $\operatorname{CCSD}(\mathrm{T}-n)$ and $\operatorname{CCSD}(\mathrm{TQ}-n)$ perturbation series and alternative approaches based on EOM-CC theory, i.e., the $\mathrm{CC}(2) \mathrm{PT}(n)$ series and the corrections of biorthogonal MMCC theory. Finally, a summary and conclusions are presented in Sec. IV.

\section{PERTURBATIVE TRIPLES AND QUADRUPLES CORRECTIONS TO THE CCSD ENERGY}

In the present section, CC energy Lagrangians are used for determining perturbative corrections to the CCSD energy, which constitute perturbation series that converge towards the CCSDT or CCSDTQ energies, depending on the target energy used in the derivation. Section II A summarizes the coupled cluster energy Lagrangian technique, while Sec. II B describes how the CCSDTQ Lagrangian may be parametrized by considering the CCSD state as the expansion point and using a Møller-Plesset partitioning of the Hamiltonian. The perturbation expansion of the CCSDTQ energy is derived in Sec. II C, from which combined triples and quadruples corrections to the CCSD energy are determined that converge towards the CCSDTQ energy in orders of the fluctuation potential. In Sec. II C, analogous triples-only corrections to the CCSD energy are also determined by retaining only those terms in the energy corrections that exclusively reference triple excitations. These corrections constitute the lowest-order corrections of a series that converges towards the CCSDT energy.

\section{A. The coupled cluster energy Lagrangian technique}

The CC Schrödinger equation may in the similaritytransformed form be expressed as

$$
\exp (-\hat{T}) \hat{H} \exp (\hat{T})|\mathrm{HF}\rangle=E_{\mathrm{CC}}|\mathrm{HF}\rangle,
$$

where $\hat{H}$ is the electronic Hamiltonian, $\hat{T}=\sum_{i} \sum_{\mu_{i}} t_{\mu_{i}} \hat{\tau}_{\mu_{i}}$ the cluster operator, and $|\mathrm{HF}\rangle$ a single determinant HartreeFock reference state. We reserve greek indices with subscripts, $\left\{\mu_{i}, v_{j}\right\}$, for denoting specific excitations, $\{\mu, v\}$, within manifolds at excitation levels $i, j, k, l$.

Projecting Eq. (2.1) against the Hartree-Fock state, $\langle\mathrm{HF}|$, gives the $\mathrm{CC}$ energy

$$
\langle\mathrm{HF}|\exp (-\hat{T}) \hat{H} \exp (\hat{T})| \mathrm{HF}\rangle=\langle\mathrm{HF}|\hat{H} \exp (\hat{T})| \mathrm{HF}\rangle=E_{\mathrm{CC}}
$$

whereas projection against the $\mu_{i}$-th excitation, $\left\langle\mu_{i}\right|$ $=\langle\mathrm{HF}| \hat{\tau}_{\mu_{i}}^{\dagger}$, gives the linked CC amplitude equations

$$
\left\langle\mu_{i}|\exp (-\hat{T}) \hat{H} \exp (\hat{T})| \mathrm{HF}\right\rangle=0 .
$$

The CC energy in Eq. (2.2) is not variational with respect to the cluster amplitudes. To obtain an energy expression that is variational, we introduce the CC Lagrangian, in which the amplitude equations are added to the energy as constraints, coupled via undetermined Lagrange multipliers, $\left\{\bar{t}_{\mu_{i}}\right\} .{ }^{37}$ This gives the CC Lagrangian

$$
\begin{aligned}
L(\mathbf{t}, \overline{\mathbf{t}})= & \langle\mathrm{HF}|\exp (-\hat{T}) \hat{H} \exp (\hat{T})| \mathrm{HF}\rangle \\
& +\sum_{i} \sum_{\mu_{i}} \bar{t}_{\mu_{i}}\left\langle\mu_{i}|\exp (-\hat{T}) \hat{H} \exp (\hat{T})| \mathrm{HF}\right\rangle .
\end{aligned}
$$

The multipliers are determined such that the Lagrangian is variational in all parameters, i.e., the cluster amplitudes and multipliers are determined from the amplitude and multiplier equations

$$
\begin{gathered}
\frac{\partial L}{\partial \bar{t}_{\mu_{i}}}=\left\langle\mu_{i}|\exp (-\hat{T}) \hat{H} \exp (\hat{T})| \mathrm{HF}\right\rangle \\
=\left\langle\mu_{i}\left|\hat{H}^{\hat{T}}\right| \mathrm{HF}\right\rangle=0, \\
\frac{\partial L}{\partial t_{\mu_{i}}}=\left\langle\mathrm{HF}\left|\exp (-\hat{T})\left[\hat{H}, \hat{\tau}_{\mu_{i}}\right] \exp (\hat{T})\right| \mathrm{HF}\right\rangle \\
+\sum_{j} \sum_{v_{j}} \bar{t}_{v_{j}}\left\langle v_{j}\left|\exp (-\hat{T})\left[\hat{H}, \hat{\tau}_{\mu_{i}}\right] \exp (\hat{T})\right| \mathrm{HF}\right\rangle \\
=\left\langle\mathrm{HF}\left|\left[\hat{H}^{\hat{T}}, \hat{\tau}_{\mu_{i}}\right]\right| \mathrm{HF}\right\rangle+\sum_{j}\left\langle\bar{t}_{j}\left|\left[\hat{H}^{\hat{T}}, \hat{\tau}_{\mu_{i}}\right]\right| \mathrm{HF}\right\rangle=0 .
\end{gathered}
$$

To obtain Eq. (2.5b), we have used the following relationship:

$$
\frac{\partial \hat{H}^{\hat{T}}}{\partial t_{\mu_{i}}}=\left[\hat{H}^{\hat{T}}, \hat{\tau}_{\mu_{i}}\right],
$$

where we have introduced the notation

$$
\hat{H}^{\hat{T}}=\exp (-\hat{T}) \hat{H} \exp (\hat{T})
$$


for the similarity-transformed Hamiltonian as well as a shorthand notation for the multiplier state, $\left\langle\bar{t}_{j}\right|$

$$
\left\langle\bar{t}_{j}\right|=\sum_{v_{j}} \bar{t}_{v_{j}}\left\langle v_{j}\right| .
$$

The Lagrangian in Eq. (2.4) is bilinear in the errors in the cluster amplitudes and multipliers.

In order to derive perturbation expansions of the $\mathrm{CC}$ energy in orders of the fluctuation potential, we introduce a Møller-Plesset partitioning of the Hamiltonian ${ }^{38}$

$$
\hat{H}=\hat{f}+\hat{\Phi},
$$

where, assuming a canonical Hartree-Fock basis, the Fock operator, $\hat{f}$, may be expressed as $\hat{f}=\sum_{P} \epsilon_{P} a_{P}^{\dagger} a_{P}$, where $\epsilon_{P}$ is the orbital energy of the generic $P$-th spin orbital. $\hat{\Phi}$ denotes the fluctuation potential, $\hat{\Phi}=\hat{g}-\hat{V}_{\mathrm{HF}}$, where $\hat{g}$ is the two-electron repulsion operator and $\hat{V}_{\mathrm{HF}}$ $=\sum_{P Q} V_{P Q} a_{P}^{\dagger} a_{Q}$ is the Fock potential with elements $V_{P Q}$ $=\sum_{I} g_{P Q I I}^{a}$. Here, $g_{P Q R S}^{a}=g_{P Q R S}-g_{P S R Q}$ is an antisymmetrized two-electron integral over spin orbitals written in Mulliken notation, ${ }^{38}$ and the set $\{P, Q, R, S\}$ denotes spin orbitals of unspecified occupation, while the index $I$ in the expression for the Fock potential refers to an occupied spin orbital. In Eq. (2.9), we have omitted $h_{\text {nuc }}$, which describes the electron-independent contribution to the energy from the nuclei.

The similarity-transformed Hamiltonian in Eq. (2.7) may be evaluated from a Baker-Campbell-Hausdorff $(\mathrm{BCH})$ expansion $^{38}$

$$
\exp (-\hat{T}) \hat{H} \exp (\hat{T})=\hat{H}+[\hat{H}, \hat{T}]+\frac{1}{2}[[\hat{H}, \hat{T}], \hat{T}]+\cdots
$$

noticing that the expansion of the similarity-transformed Fock operator in Eq. (2.9) terminates after two terms

$$
\hat{f}^{\hat{T}}=\hat{f}+\sum_{i} \sum_{\mu_{i}} t_{\mu_{i}} \epsilon_{\mu_{i}} \hat{\tau}_{\mu_{i}}
$$

as all nested (i.e., double, triple, etc.) commutators between the Fock operator and cluster operators vanish, since all cluster operators commute. For $\hat{\Phi}^{\hat{T}}$, on the other hand, the $\mathrm{BCH}$ expansion truncates after the fifth term. In general, $\epsilon_{\mu_{i}}$ designates the difference in orbital energies between the virtual and occupied spin orbitals of excitation $\mu_{i}$. We note that in the case of non-canonical or non-HF orbitals, the similaritytransformed Fock operator contains additional terms. However, the approach described in the following can be easily generalized to cope with these problems in a way similar to that followed in Ref. 8, which handled the case of $\operatorname{CCSD}(\mathrm{T})$.

\section{B. Parametrization of the CCSDTQ Lagrangian around a CCSD reference point}

In the CCSDTQ ${ }^{26}$ model (denoted with a shorthand notation by a TQ subscript), the cluster operator, $\hat{T}$, is truncated at the level of quadruple excitations, $\hat{T}=\sum_{i=1}^{4} \sum_{\mu_{i}} t_{\mu_{i}} \hat{\tau}_{\mu_{i}}$, giving variational parameters, $\{\mathbf{t}, \overline{\mathbf{t}}\}$, at all excitation levels ranging from singles to quadruples. Hence, we may write the
CCSDTQ Lagrangian in Eq. (2.4) as follows:

$$
L_{\mathrm{TQ}}(\mathbf{t}, \overline{\mathbf{t}})=\left\langle\mathrm{HF}\left|\hat{H}^{\hat{T}}\right| \mathrm{HF}\right\rangle+\sum_{i=1}^{4}\left\langle\bar{t}_{i}\left|\hat{H}^{\hat{T}}\right| \mathrm{HF}\right\rangle .
$$

The CCSDTQ Lagrangian in Eq. (2.12) may be recasted by parametrizing it around the CCSD reference energy point. In essence, this alternative, but analogous parametrization divides the cluster operator, $\hat{T}$, at the CCSD expansion point into a CCSD component, $\left\{{ }^{*} \hat{T}_{1},{ }^{*} \hat{T}_{2}\right\}$, which has been determined from a CCSD calculation, and a component, $\delta \hat{T}$ $=\sum_{k=1}^{4} \delta \hat{T}_{k}$ (where $\delta \hat{T}_{k}=\sum_{\mu_{k}} \delta t_{\mu_{k}} \hat{\tau}_{\mu_{k}}$ ), which is to be determined. Similarly, the Lagrange multipliers consist of a CCSD component, $\left\{{ }^{*} \bar{t}_{\mu_{1}},{ }^{*} \bar{t}_{\mu_{2}}\right\}$, that has been determined from the CCSD calculation and a set, $\left\{\delta \bar{\mu}_{\mu_{k}}\right\}$ (in the range $k=1-4$ ), which is to be determined. By parametrizing $L_{\mathrm{TQ}}$ around the CCSD energy point, all $\left\{\delta t_{\mu_{i}}, \delta \bar{t}_{\mu_{i}}\right\}$ parameters for $i=1,2$ describe corrections in the singles and doubles spaces relative to the CCSD values, $\left\{{ }^{*} t_{\mu_{i}}, * \bar{t}_{\mu_{i}}\right\}$. For $i=3,4$, the parameters, $\left\{\delta t_{\mu_{i}}, \delta \bar{t}_{\mu_{i}}\right\}$, parametrize the full triples and quadruples spaces, which, naturally, have no CCSD component. Introducing the above parameters into Eq. (2.12), the CCSDTQ Lagrangian parametrized around the CCSD reference energy point may be expressed as

$$
\begin{aligned}
L_{\mathrm{TQ}}\left({ }^{*} \mathbf{t},{ }^{*} \overline{\mathbf{t}}, \delta \mathbf{t}, \delta \overline{\mathbf{t}}\right) & \\
= & \left\langle\mathrm{HF}\left|\exp (-\delta \hat{T}) \hat{H}^{*} \hat{T} \exp (\delta \hat{T})\right| \mathrm{HF}\right\rangle \\
& +\sum_{i=1}^{2}\left\langle{ }^{*} \bar{t}_{i}+\delta \bar{t}_{i}\left|\exp (-\delta \hat{T}) \hat{H}^{*} \hat{T} \exp (\delta \hat{T})\right| \mathrm{HF}\right\rangle \\
& +\sum_{j=3}^{4}\left\langle\delta \bar{t}_{j}\left|\exp (-\delta \hat{T}) \hat{H}^{*} \hat{T} \exp (\delta \hat{T})\right| \mathrm{HF}\right\rangle .
\end{aligned}
$$

In Eq. (2.13), we have introduced the CCSD similaritytransformed Hamiltonian

$$
\hat{H}^{*} \hat{T} \equiv \exp \left(-\left({ }^{*} \hat{T}_{1}+{ }^{*} \hat{T}_{2}\right)\right) \hat{H} \exp \left({ }^{*} \hat{T}_{1}+{ }^{*} \hat{T}_{2}\right) .
$$

We note that the $\mathrm{CCSDT}^{25}$ Lagrangian parametrized around the CCSD reference energy point is obtained by limiting $\delta \hat{T}$, and thus the $\delta t_{\mu_{k}}$ amplitudes and $\delta \bar{t}_{\mu_{k}}$ multipliers, to the range $k=1-3$ in Eq. (2.13).

The CCSD amplitudes and Lagrange multipliers satisfy the CCSD amplitude and multiplier equations

$$
\begin{gathered}
\left\langle\mu_{i}\left|\hat{H}^{*} \hat{T}\right| \mathrm{HF}\right\rangle=0, \\
\left\langle\mathrm{HF}\left|\left[\hat{H}^{*} \hat{T}, \hat{\tau}_{\mu_{i}}\right]\right| \mathrm{HF}\right\rangle+\sum_{j=1}^{2}\left\langle{ }^{*} \bar{t}_{j}\left|\left[\hat{H}^{*} \hat{T}, \hat{\tau}_{\mu_{i}}\right]\right| \mathrm{HF}\right\rangle=0,
\end{gathered}
$$

with $i=1,2$. The CCSD Lagrangian is given as

$$
L_{\mathrm{CCSD}}=\left\langle\mathrm{HF}\left|\hat{H}^{*} \hat{T}\right| \mathrm{HF}\right\rangle+\sum_{i=1}^{2}\left\langle{ }^{*} \bar{t}_{i}\left|\hat{H}^{*} \hat{T}\right| \mathrm{HF}\right\rangle .
$$

Since the CCSD amplitudes satisfy the amplitude equation in (2.15a), the last term in Eq. (2.16) vanishes, but it is kept here to make the analysis to follow more transparent. 
The CCSDTQ Lagrangian expanded around the CCSD reference energy point may be simplified by introducing $L_{\mathrm{CCSD}}$ into the Lagrangian and making use of the fact that the CCSD amplitudes and multipliers satisfy Eqs. (2.15a) and (2.15b). Carrying out a $\mathrm{BCH}$ expansion in Eq. (2.13), we obtain

$$
\begin{aligned}
& L_{\mathrm{TQ}}\left({ }^{*} \mathbf{t},{ }^{*} \overline{\mathbf{t}}, \delta \mathbf{t}, \delta \overline{\mathbf{t}}\right) \\
& =\langle\operatorname{HF}|\left(\hat{H}^{*} \hat{T}+\left[\hat{H}^{*} \hat{T}, \delta \hat{T}\right]\right. \\
& \left.+\frac{1}{2}\left[\left[\hat{H}^{*} \hat{T}, \delta \hat{T}\right], \delta \hat{T}\right]+\cdots\right)|\mathrm{HF}\rangle \\
& +\sum_{i=1}^{2}\left\langle{ }^{*} \bar{t}_{i}\right|\left(\hat{H}^{*} \hat{T}+\left[\hat{H}^{*} \hat{T}, \delta \hat{T}\right]\right. \\
& \left.+\frac{1}{2}\left[\left[\hat{H}^{*} \hat{T}, \delta \hat{T}\right], \delta \hat{T}\right]+\cdots\right)|\mathrm{HF}\rangle \\
& +\sum_{j=1}^{4}\left\langle\delta \bar{t}_{j}\right|\left(\hat{H}^{*} \hat{T}+\left[\hat{H}^{*} \hat{T}, \delta \hat{T}\right]\right. \\
& \left.+\frac{1}{2}\left[\left[\hat{H}^{*} \hat{T}, \delta \hat{T}\right], \delta \hat{T}\right]+\cdots\right)|\mathrm{HF}\rangle .
\end{aligned}
$$

The CCSD Lagrangian in Eq. (2.16) may be identified from the $\hat{H}^{*} \hat{T}$ contribution to the first two terms of Eq. (2.17). For the $\left[\hat{H}^{*} \hat{T}, \delta \hat{T}\right]$ commutator contributions to the first two terms, we get

$$
\begin{gathered}
\left\langle\mathrm{HF}\left|\left[\hat{H}^{*} \hat{T}, \delta \hat{T}\right]\right| \mathrm{HF}\right\rangle+\sum_{i=1}^{2}\left\langle{ }^{*} \bar{t}_{i}\left|\left[\hat{H}^{*} \hat{T}, \delta \hat{T}\right]\right| \mathrm{HF}\right\rangle \\
=\sum_{j=1}^{2} \sum_{v_{j}} \delta t_{v_{j}}\left\{\left\langle\mathrm{HF}\left|\left[\hat{H}^{*} \hat{T}^{2}, \hat{\tau}_{v_{j}}\right]\right| \mathrm{HF}\right\rangle\right. \\
\left.+\sum_{i=1}^{2}\left\langle{ }^{*} \bar{t}_{i}\left|\left[\hat{H}^{*} \hat{T}, \hat{\tau}_{v_{j}}\right]\right| \mathrm{HF}\right\rangle\right\} \\
+\sum_{i=1}^{2} \sum_{k=3}^{4}\left\langle{ }^{*} \bar{t}_{i}\left|\left[\hat{\Phi}^{*} \hat{T}, \delta \hat{T}_{k}\right]\right| \mathrm{HF}\right\rangle \\
=\sum_{i=1}^{2} \sum_{k=3}^{4}\left\langle{ }^{*} \bar{t}_{i}\left|\left[\hat{\Phi}^{*} \hat{T}, \delta \hat{T}_{k}\right]\right| \mathrm{HF}\right\rangle,
\end{gathered}
$$

where $\hat{\Phi}^{*} \hat{T}$ is defined analogously to Eq. (2.14). To obtain the first equality in Eq. (2.18), we have used that the contributions from the $\delta \hat{T}_{3}$ and $\delta \hat{T}_{4}$ operators vanish for the first term due to the excitation rank of the operators, while the last equality is obtained by virtue of Eq. (2.15b) for both the single and double excitations. Furthermore, the $\delta \bar{t}_{1}$ and $\delta \bar{t}_{2}$ contributions to Eq. (2.17), which involve $\hat{H}^{*} \hat{T}$, will vanish, as the corresponding terms satisfy the CCSD amplitude equations. Finally, the $\mathrm{HF}$ expectation value in the first line of Eq. (2.17) consists, apart from the zeroth-order contribution, of a term quadratic in the singles correction. Employing the above findings, we arrive at the following simplified expression for the CCSDTQ Lagrangian:

$$
\begin{aligned}
& L_{\mathrm{TQ}}\left({ }^{*} \mathbf{t},{ }^{*} \overline{\mathbf{t}}, \delta \mathbf{t}, \delta \overline{\mathbf{t}}\right) \\
& =L_{\mathrm{CCSD}}+\sum_{l=1}^{4} \delta \bar{t}_{\mu_{l}} \epsilon_{\mu_{l}} \delta t_{\mu_{l}}+\frac{1}{2}\left\langle\mathrm{HF}\left|\left[\left[\hat{\Phi}, \delta \hat{T}_{1}\right], \delta \hat{T}_{1}\right]\right| \mathrm{HF}\right\rangle \\
& +\sum_{i=1}^{2}\left\{\sum_{k=3}^{4}\left\langle^{*} \bar{t}_{i}\left|\left[\hat{\Phi}^{*} \hat{T}, \delta \hat{T}_{k}\right]\right| \mathrm{HF}\right\rangle\right. \\
& \left.\quad+\left\langle^{*} \bar{t}_{i}\left|\left(\frac{1}{2}\left[\left[\hat{\Phi}^{*} \hat{T}, \delta \hat{T}\right], \delta \hat{T}\right]+\cdots\right)\right| \mathrm{HF}\right\rangle\right\} \\
& +\sum_{j=1}^{2}\left\langle\delta \bar{t}_{j}\left|\left(\left[\hat{\Phi}^{*} \hat{T}, \delta \hat{T}\right]+\frac{1}{2}\left[\left[\hat{\Phi}^{*} \hat{T}, \delta \hat{T}\right], \delta \hat{T}\right]+\cdots\right)\right| \mathrm{HF}\right\rangle \\
& +\sum_{k=3}^{4}\left\langle\delta \bar{t}_{k}\right|\left(\hat{\Phi}^{*} \hat{T}+\left[\hat{\Phi}^{*} \hat{T}, \delta \hat{T}\right]\right. \\
& \left.\quad+\frac{1}{2}\left[\left[\hat{\Phi}^{*} \hat{T}, \delta \hat{T}\right], \delta \hat{T}\right]+\cdots\right)|\mathrm{HF}\rangle, \quad(2.19)
\end{aligned}
$$

where we have used Eq. (2.11) for all of the commutators between $\hat{f}^{*} \hat{T}$ and $\delta \hat{T}_{l}(l=1-4)$ to arrive at an expression that only references the CCSD similarity-transformed fluctuation potential.

The Lagrangian in Eq. (2.19) is variational in the cluster amplitudes and multipliers and differentiation with respect to the multipliers thus gives the amplitude equations for all the involved excitation levels

$$
\begin{gathered}
\epsilon_{\mu_{1}} \delta t_{\mu_{1}}+\left\langle\mu_{1}\left|\left(\left[\hat{\Phi}^{*} \hat{T}, \delta \hat{T}\right]+\frac{1}{2}\left[\left[\hat{\Phi}^{*} \hat{T}, \delta \hat{T}\right], \delta \hat{T}\right]+\cdots\right)\right| \mathrm{HF}\right\rangle=0, \\
(2.20 \mathrm{a}) \\
\epsilon_{\mu_{2}} \delta t_{\mu_{2}}+\left\langle\mu_{2}\left|\left(\left[\hat{\Phi}^{*} \hat{T}, \delta \hat{T}\right]+\frac{1}{2}\left[\left[\hat{\Phi}^{*} \hat{T}, \delta \hat{T}\right], \delta \hat{T}\right]+\cdots\right)\right| \mathrm{HF}\right\rangle=0, \\
(2.20 \mathrm{~b}) \\
\epsilon_{\mu_{3}} \delta t_{\mu_{3}}+\left\langle\mu_{3}\right|\left(\hat{\Phi}^{*} \hat{T}+\left[\hat{\Phi}^{*} \hat{T}, \delta \hat{T}\right]\right. \\
\left.+\frac{1}{2}\left[\left[\hat{\Phi}^{*} \hat{T}, \delta \hat{T}\right], \delta \hat{T}\right]+\cdots\right)|\mathrm{HF}\rangle=0, \\
\epsilon_{\mu_{4}} \delta t_{\mu_{4}}+\left\langle\mu_{4}\right|\left(\hat{\Phi}^{*} \hat{T}+\left[\hat{\Phi}^{*} \hat{T}, \delta \hat{T}\right]\right. \\
\left.+\frac{1}{2}\left[\left[\hat{\Phi}^{*} \hat{T}, \delta \hat{T}\right], \delta \hat{T}\right]+\cdots\right)|\mathrm{HF}\rangle=0,
\end{gathered}
$$

while differentiation with respect to the amplitudes gives the corresponding multipliers for the different excitation levels

$$
\begin{aligned}
\epsilon_{\mu_{1}} \delta \bar{t}_{\mu_{1}} & +\left\langle\mathrm{HF}\left|\left[\left[\hat{\Phi}, \hat{\tau}_{\mu_{1}}\right], \delta \hat{T}_{1}\right]\right| \mathrm{HF}\right\rangle \\
& +\sum_{i=1}^{2} \sum_{k=1}^{3}\left\langle{ }^{*} \bar{t}_{i}\left|\left(\left[\left[\hat{\Phi}^{*} \hat{T}, \hat{\tau}_{\mu_{1}}\right], \delta \hat{T}_{k}\right]+\cdots\right)\right| \mathrm{HF}\right\rangle \\
& +\sum_{j=1}^{4}\left\langle\delta \bar{t}_{j}\right|\left(\left[\hat{\Phi}^{*} \hat{T}, \hat{\tau}_{\mu_{1}}\right]\right. \\
& \left.+\left[\left[\hat{\Phi}^{*} \hat{T}, \hat{\tau}_{\mu_{1}}\right], \delta \hat{T}\right]+\cdots\right)|\mathrm{HF}\rangle=0,
\end{aligned}
$$




$$
\begin{aligned}
& \epsilon_{\mu_{2}} \delta \bar{t}_{\mu_{2}}+\sum_{i=1}^{2} \sum_{k=1}^{2}\left\langle{ }^{*} \bar{t}_{i}\left|\left(\left[\left[\hat{\Phi}^{*} \hat{T}, \hat{\tau}_{\mu_{2}}\right], \delta \hat{T}_{k}\right]+\cdots\right)\right| \mathrm{HF}\right\rangle \\
& +\sum_{j=1}^{4}\left\langle\delta \bar{t}_{j}\right|\left(\left[\hat{\Phi}^{*} \hat{T}, \hat{\tau}_{\mu_{2}}\right]\right. \\
& \left.+\left[\left[\hat{\Phi}^{*} \hat{T}, \hat{\tau}_{\mu_{2}}\right], \delta \hat{T}\right]+\ldots\right)|\mathrm{HF}\rangle=0, \\
& \epsilon_{\mu_{3}} \delta \bar{t}_{\mu_{3}}+\sum_{i=1}^{2}\left\langle{ }^{*} \bar{t}_{i}\left|\left(\left[\hat{\Phi}^{*} \hat{T}, \hat{\tau}_{\mu_{3}}\right]+\left[\left[\hat{\Phi}^{*} \hat{T}, \hat{\tau}_{\mu_{3}}\right], \delta \hat{T}_{1}\right]\right)\right| \mathrm{HF}\right\rangle \\
& +\sum_{j=1}^{4} \sum_{k=1}^{3}\left\langle\delta \bar{t}_{j}\right|\left(\left[\hat{\Phi}^{*} \hat{T}, \hat{\tau}_{\mu_{3}}\right]\right. \\
& \left.+\left[\left[\hat{\Phi}^{*} \hat{T}, \hat{\tau}_{\mu_{3}}\right], \delta \hat{T}_{k}\right]+\cdots\right)|\mathrm{HF}\rangle=0, \\
& \epsilon_{\mu_{4}} \delta \bar{t}_{\mu_{4}}+\sum_{i=1}^{2}\left\langle{ }^{*} \bar{t}_{i}\left|\left[\hat{\Phi}^{*} \hat{T}, \hat{\tau}_{\mu_{4}}\right]\right| \mathrm{HF}\right\rangle \\
& +\sum_{j=1}^{4} \sum_{k=1}^{2}\left\langle\delta \bar{t}_{j}\right|\left(\left[\hat{\Phi}^{*} \hat{T}, \hat{\tau}_{\mu_{4}}\right]\right. \\
& \left.+\left[\left[\hat{\Phi}^{*} \hat{T}, \hat{\tau}_{\mu_{4}}\right], \delta \hat{T}_{k}\right]+\cdots\right)|\mathrm{HF}\rangle=0 .
\end{aligned}
$$

To obtain Eqs. (2.21a) and (2.21b), we have made use of Eq. (2.15b).

Equations (2.19)-(2.21d) describe a parametrization of the CCSDTQ Lagrangian, amplitude, and multiplier equations for an expansion around a CCSD reference energy point, in which the equations are fully simplified due to fact that the CCSD parameters satisfy the CCSD cluster amplitude and multiplier equations. Furthermore, a Møller-Plesset partitioning of the Hamiltonian is performed, making the parametrization well-suited for determining perturbative corrections to the CCSD energy in orders of the fluctuation potential that converge towards the CCSDTQ energy.

\section{The CCSDTQ Lagrangian expanded in orders of the fluctuation potential}

We will now carry out a perturbation analysis in orders of the fluctuation potential for the CCSDTQ Lagrangian in Eq. (2.19). We thus expand the Lagrangian, the cluster amplitudes, and the multipliers in orders of the fluctuation potential as

$$
\begin{gathered}
L_{\mathrm{TQ}}=L_{\mathrm{TQ}}^{(0)}+L_{\mathrm{TQ}}^{(1)}+L_{\mathrm{TQ}}^{(2)}+\cdots, \\
\delta t_{\mu}=\delta t_{\mu}^{(0)}+\delta t_{\mu}^{(1)}+\delta t_{\mu}^{(2)}+\cdots, \\
\delta \bar{t}_{\mu}=\delta \bar{t}_{\mu}^{(0)}+\delta \bar{t}_{\mu}^{(1)}+\delta \bar{t}_{\mu}^{(2)}+\cdots,
\end{gathered}
$$

where orders in the fluctuation potential are designated inside parentheses and $L_{\mathrm{TQ}}$ converges to the CCSDTQ energy. We denote the series in Eq. (2.22a) as the CCSD(TQ-n) series, referencing that the expansion energy point is that for a CCSD parent model, the target energy is the CCSDTQ energy, and that the series is obtained using coupled cluster Lagrangian perturbation theory to order $n$. Similarly, when the target energy is the CCSDT energy, we will refer to the resulting series in CC Lagrangian perturbation theory as the $\operatorname{CCSD}(\mathrm{T}-n)$ series, see the discussion below Eq. (2.14).

From the structure of the amplitude and multiplier equations in Eqs. (2.20a)-(2.21d), it is seen that both the zerothorder amplitudes and multipliers for excitation level one through four vanish

$$
\delta t_{\mu_{j}}^{(0)}=\delta \bar{t}_{\mu_{j}}^{(0)}=0, \quad j=1-4 .
$$

In the $\mathrm{CCSD}(\mathrm{TQ}-n)$ (and similarly in the $\operatorname{CCSD}(\mathrm{T}-n)$ ) series, all zeroth-order parameters are thus zero and the zeroth-order Lagrangian is given as

$$
L_{\mathrm{T}}^{(0)}=L_{\mathrm{TQ}}^{(0)}=L_{\mathrm{CCSD}} .
$$

We are interested in determining energy corrections to the CCSD energy through fourth order in the fluctuation potential. Applying the $2 n+1$ and $2 n+2$ rules $^{39}$ for the cluster amplitudes and multipliers, respectively, we recognize that only amplitudes and multipliers to first order in the fluctuation potential, $\delta t_{\mu}^{(1)}, \delta \bar{t}_{\mu}^{(1)}$, will contribute to energy corrections through third order, while second-order amplitudes, $\delta t_{\mu}^{(2)}$, in addition to first-order amplitudes and multipliers, $\delta t_{\mu}^{(1)}, \delta \bar{t}_{\mu}^{(1)}$, are needed for obtaining the fourth-order energy correction. We therefore need only to determine the first and second-order amplitudes and first-order multipliers from Eqs. (2.20a)-(2.20d) and (2.21a)-(2.21d), respectively. Since we know that the leading order of $\delta t_{\mu_{k}}$ for the range $k=1-$ 4 is at least one, cf. Eq. (2.23), we may write the amplitude equations in Eqs. (2.20a)-(2.20d) as

$$
\begin{aligned}
& \epsilon_{\mu_{1}} \delta t_{\mu_{1}}+\sum_{i=1}^{3}\left\langle\mu_{1}\left|\left[\hat{\Phi}^{*} \hat{T}, \delta \hat{T}_{i}\right]\right| \mathrm{HF}\right\rangle+\mathcal{O}(3)=0, \\
& \epsilon_{\mu_{2}} \delta t_{\mu_{2}}+\sum_{i=1}^{4}\left\langle\mu_{2}\left|\left[\hat{\Phi}^{*} \hat{T}, \delta \hat{T}_{i}\right]\right| \mathrm{HF}\right\rangle+\mathcal{O}(3)=0, \\
& \epsilon_{\mu_{3}} \delta t_{\mu_{3}}+\left\langle\mu_{3}\left|\hat{\Phi}^{*} \hat{T}\right| \mathrm{HF}\right\rangle+\sum_{i=3}^{4}\left\langle\mu_{3}\left|\left[\hat{\Phi}^{*} \hat{T}, \delta \hat{T}_{i}\right]\right| \mathrm{HF}\right\rangle+\mathcal{O}(3)=0,
\end{aligned}
$$

$\epsilon_{\mu_{4}} \delta t_{\mu_{4}}+\left\langle\mu_{4}\left|\hat{\Phi}^{*} \hat{T}\right| \mathrm{HF}\right\rangle+\sum_{i=3}^{4}\left\langle\mu_{4}\left|\left[\hat{\Phi}^{*} \hat{T}, \delta \hat{T}_{i}\right]\right| \mathrm{HF}\right\rangle+\mathcal{O}(3)=0$,

where we have introduced a $\mathcal{O}(p)$ nomenclature for denoting terms of order $p$ in the fluctuation potential. From Eqs. (2.25a) and (2.25b), it is seen that the leading order of the singles and doubles amplitudes is two. Since only triples and quadruples amplitudes enter in first order, the summations in the last terms of Eqs. (2.25c) and (2.25d) have been restricted to include only triple and quadruple excitations. The amplitude equations in Eqs. (2.25a)-(2.25d) allow us to express the first-order amplitudes as

$$
\begin{aligned}
& \delta t_{\mu_{3}}^{(1)}=-\epsilon_{\mu_{3}}^{-1}\left\langle\mu_{3}\left|\hat{\Phi}^{*} \hat{T}\right| \mathrm{HF}\right\rangle, \\
& \delta t_{\mu_{4}}^{(1)}=-\epsilon_{\mu_{4}}^{-1}\left\langle\mu_{4}\left|\hat{\Phi}^{*} \hat{T}\right| \mathrm{HF}\right\rangle,
\end{aligned}
$$


and the second-order amplitudes as

$$
\begin{gathered}
\delta t_{\mu_{1}}^{(2)}=-\epsilon_{\mu_{1}}^{-1}\left\langle\mu_{1}\left|\left[\hat{\Phi}^{*} \hat{T}, \delta \hat{T}_{3}^{(1)}\right]\right| \mathrm{HF}\right\rangle, \\
\delta t_{\mu_{2}}^{(2)}=-\epsilon_{\mu_{2}}^{-1} \sum_{i=3}^{4}\left\langle\mu_{2}\left|\left[\hat{\Phi}^{*} \hat{T}, \delta \hat{T}_{i}^{(1)}\right]\right| \mathrm{HF}\right\rangle, \\
\delta t_{\mu_{3}}^{(2)}=-\epsilon_{\mu_{3}}^{-1} \sum_{i=3}^{4}\left\langle\mu_{3}\left|\left[\hat{\Phi}^{*} \hat{T}, \delta \hat{T}_{i}^{(1)}\right]\right| \mathrm{HF}\right\rangle, \\
\delta t_{\mu_{4}}^{(2)}=-\epsilon_{\mu_{4}}^{-1} \sum_{i=3}^{4}\left\langle\mu_{4}\left|\left[\hat{\Phi}^{*} \hat{T}, \delta \hat{T}_{i}^{(1)}\right]\right| \mathrm{HF}\right\rangle,
\end{gathered}
$$

where $\delta \hat{T}_{i}^{(1)}=\sum_{\mu_{i}} \delta t_{\mu_{i}}^{(1)} \hat{\tau}_{\mu_{i}}$. Evidently, when the target energy is the CCSDT rather than the CCSDTQ energy, the effect from quadruple excitations are omitted from the firstand second-order amplitudes in Eqs. (2.26a), (2.26b), and (2.27b)-(2.27d). This implies that in the $\operatorname{CCSD}(\mathrm{T}-n)$ series, the first-order triples amplitudes are those given by Eq. (2.26a), while the second-order singles, doubles, and triples amplitudes are given by Eqs. (2.27a)-(2.27c), however, with no reference to the quadruple excitations. Consequently, these amplitudes are changed in moving from the $\operatorname{CCSD}(\mathrm{T}-n)$ to the $\operatorname{CCSD}(\mathrm{TQ}-n)$ series.

Turning the attention now to the singles and doubles multipliers in Eqs. (2.21a) and (2.21b), we recognize that the leading order of both sets of multipliers will be two

$$
\begin{aligned}
& \delta \bar{t}_{\mu_{1}}=\delta \bar{t}_{\mu_{1}}^{(2)}+\mathcal{O}(3), \\
& \delta \bar{t}_{\mu_{2}}=\delta \bar{t}_{\mu_{2}}^{(2)}+\mathcal{O}(3) .
\end{aligned}
$$

As we are only interested in analytical expressions for energy corrections through fourth order, second-order Lagrange multipliers are not needed, and the second-order singles and doubles multipliers will therefore not be detailed any further. Considering finally the determination of the triples and quadruples Lagrange multipliers in Eqs. (2.21c) and (2.21d), we explicitly retain only terms up to first order in the fluctuation potential, giving

$$
\begin{gathered}
\delta \bar{t}_{\mu_{3}}^{(1)}=-\epsilon_{\mu_{3}}^{-1} \sum_{i=1}^{2}\left\langle^{*} \bar{t}_{i}\left|\left[\hat{\Phi}^{*} \hat{T}^{2}, \hat{\tau}_{\mu_{3}}\right]\right| \mathrm{HF}\right\rangle, \\
\delta \bar{t}_{\mu_{4}}^{(1)}=-\epsilon_{\mu_{4}}^{-1}\left\langle^{*} \bar{t}_{2}\left|\left[\hat{\Phi}^{*} \hat{T}, \hat{\tau}_{\mu_{4}}\right]\right| \mathrm{HF}\right\rangle .
\end{gathered}
$$

We are now capable of evaluating the corrections to the CCSD energy from the Lagrangian in Eq. (2.19). We will use a $E^{(p)}$ nomenclature for the energy correction to $p$-th order when this has been obtained from $L^{(p)}$ by the application of Wigner's rules. Using the $2 n+1$ rule for the amplitudes and the $2 n$ +2 rule for the multipliers, we recognize that only zerothorder amplitudes and multipliers are required for obtaining the first-order energy correction. As the zeroth-order amplitudes and multipliers vanish, cf. Eq. (2.23), we obtain

$$
E_{\mathrm{T}}^{(1)}=E_{\mathrm{TQ}}^{(1)}=0 .
$$

For the second-order energy correction, applying Wigner's rules dictates that only terms containing first-order amplitudes and zeroth-order multipliers have to be considered. From
Eq. (2.19), we thus find $E_{\mathrm{TQ}}^{(2)}$ as a sum of two terms; one originating from triple excitations, $E_{\mathrm{T}}^{(2)}$, and one from quadruple excitations, $E_{\mathrm{Q}}^{(2)}$

$$
\begin{aligned}
E_{\mathrm{TQ}}^{(2)} & =E_{\mathrm{T}}^{(2)}+E_{\mathrm{Q}}^{(2)} \\
& =\sum_{i=1}^{2}\left\langle{ }^{*} \bar{t}_{i}\left|\left[\hat{\Phi}^{*} \hat{T}, \delta \hat{T}_{3}^{(1)}\right]\right| \mathrm{HF}\right\rangle+\left\langle{ }^{*} \bar{t}_{2}\left|\left[\hat{\Phi}, \delta \hat{T}_{4}^{(1)}\right]\right| \mathrm{HF}\right\rangle .
\end{aligned}
$$

Similarly, to obtain the third-order energy correction, we need in addition to the first-order amplitudes also to consider the first-order multipliers. From Eq. (2.19), $E_{\mathrm{TQ}}^{(3)}$ may analogously to Eq. (2.31) be written as a sum of a triples-only term, $E_{\mathrm{T}}^{(3)}$, and a combined triples and quadruples term, $E_{\mathrm{Q}}^{(3)}$

$$
\begin{aligned}
E_{\mathrm{TQ}}^{(3)}= & E_{\mathrm{T}}^{(3)}+E_{\mathrm{Q}}^{(3)} \\
= & \left\langle\delta \bar{t}_{3}^{(1)}\left|\left[\hat{\Phi}^{*} \hat{T}, \delta \hat{T}_{3}^{(1)}\right]\right| \mathrm{HF}\right\rangle+\left\langle\delta \bar{t}_{3}^{(1)}\left|\left[\hat{\Phi}^{*} \hat{T}, \delta \hat{T}_{4}^{(1)}\right]\right| \mathrm{HF}\right\rangle \\
& +\left\langle\delta \bar{t}_{4}^{(1)}\left|\left[\hat{\Phi}^{*} \hat{T}, \delta \hat{T}_{3}^{(1)}\right]\right| \mathrm{HF}\right\rangle+\left\langle\delta \bar{t}_{4}^{(1)}\left|\left[\hat{\Phi}^{*} \hat{T}, \delta \hat{T}_{4}^{(1)}\right]\right| \mathrm{HF}\right\rangle .
\end{aligned}
$$

Finally, the fourth-order energy correction requires firstand second-order amplitudes and first-order multipliers. Expressing $E_{\mathrm{TQ}}^{(4)}$ as a sum of $E_{\mathrm{T}}^{(4)}$, a triples-only term, and $E_{\mathrm{Q}}^{(4)}$, a combined triples and quadruples term, it reads

$$
\begin{aligned}
E_{\mathrm{TQ}}^{(4)}= & E_{\mathrm{T}}^{(4)}+E_{\mathrm{Q}}^{(4)} \\
= & \left\langle^{*} \bar{t}_{2}\left|\left[\left[\hat{\Phi}, \delta \hat{T}_{3}^{(1)}\right], \delta \hat{T}_{1}^{(2)}\right]\right| \mathrm{HF}\right\rangle \\
& +\left\langle\delta \bar{t}_{3}^{(1)}\left|\left[\hat{\Phi}^{*} \hat{T}, \delta \hat{T}_{1}^{(2)}+\delta \hat{T}_{2}^{(2)}+\delta \hat{T}_{3}^{(2)}\right]\right| \mathrm{HF}\right\rangle \\
& +\left\langle\delta \bar{t}_{3}^{(1)}\left|\left[\hat{\Phi}^{*} \hat{T}, \delta \hat{T}_{4}^{(2)}\right]\right| \mathrm{HF}\right\rangle \\
& +\frac{1}{2}\left\langle\delta \bar{t}_{4}^{(1)}\left|\left[\left[\hat{\Phi}, \delta \hat{T}_{3}^{(1)}\right], \delta \hat{T}_{3}^{(1)}\right]\right| \mathrm{HF}\right\rangle \\
& +\left\langle\delta \bar{t}_{4}^{(1)}\left|\left[\hat{\Phi}^{*} \hat{T}, \delta \hat{T}_{1}^{(2)}+\delta \hat{T}_{2}^{(2)}+\delta \hat{T}_{3}^{(2)}+\delta \hat{T}_{4}^{(2)}\right]\right| \mathrm{HF}\right\rangle .
\end{aligned}
$$

Equations (2.31)-(2.33) constitute the $\operatorname{CCSD}(\mathrm{TQ}-2)$, CCSD(TQ-3), and CCSD(TQ-4) corrections to the CCSD energy of the CCSD(TQ- $n$ ) hierarchy. Recalling that the difference between the $\operatorname{CCSD}(\mathrm{TQ}-n)$ and $\operatorname{CCSD}(\mathrm{T}-n)$ hierarchies lies in the target energy of the series, CCSDTQ and CCSDT, respectively, the CCSD $(\mathrm{T}-n)$ corrections may readily be determined from those of the CCSD(TQ- $n$ ) hierarchy by neglecting all terms which reference quadruple excitations. Thus, $E_{\mathrm{T}}^{(2)}$ of Eq. (2.31) and $E_{\mathrm{T}}^{(3)}$ of Eq. (2.32) make up the $\operatorname{CCSD}(\mathrm{T}-2)$ and $\operatorname{CCSD}(\mathrm{T}-3)$ corrections to the CCSD energy, respectively. The $\operatorname{CCSD}(\mathrm{T}-4)$ correction may be determined from Eq. (2.33) by retaining only the $E_{\mathrm{T}}^{(4)}$ term and building the second-order amplitudes for excitation levels two and three without any reference to quadruple excitations, cf. the discussion following Eqs. (2.27a)-(2.27d). We note how energy corrections emerging from relaxation in the singles and doubles amplitudes relative to the CCSD values 
enter the $\operatorname{CCSD}(\mathrm{T}-n)$ and $\operatorname{CCSD}(\mathrm{TQ}-n)$ series at fourth order. It should be further noticed that the corrections of these two series are rigorously size extensive, term-wise even, as all involved terms are expressed in terms of commutators. ${ }^{38}$

\section{COMPARISON OF THE CCSD(TQ-n) SERIES WITH OTHER APPROACHES}

In Sec. II, we used the coupled cluster Lagrangian technique to determine perturbational corrections to the CCSD energy, while previous derivations of such energy corrections have usually been based on an EOM-CC formalism; see Sec. I for a discussion and references. In this section, we will compare the EOM-CC and Lagrangian formalisms in Secs. III A and III B and perform detailed comparisons of the $\mathrm{CCSD}(\mathrm{TQ}-n)$ series of the present work with the CC(2)PT $(n)$ series of Hirata and co-workers ${ }^{16}$ in Sec. III C. Finally, we compare our Lagrangian-based theory with the biorthogonal MMCC theory of Piecuch and co-workers ${ }^{19-21}$ in Sec. III D.

\section{A. Parametrizations in the EOM-CC and Lagrangian formalisms}

In the EOM-CC picture, ${ }^{32}$ the FCI energy may be parametrized as

$$
E_{\mathrm{FCI}}=\frac{\left\langle\mathrm{HF}\left|\hat{\mathcal{L}} \hat{H}^{*} \hat{T} \hat{\mathcal{R}}\right| \mathrm{HF}\right\rangle}{\langle\mathrm{HF}|\hat{\mathcal{L}} \hat{\mathcal{R}}| \mathrm{HF}\rangle},
$$

where $\hat{\mathcal{R}}$ and $\hat{\mathcal{L}}$ are CI-like, linear excitation and de-excitation operators containing components at all excitation levels, $N$, i.e.,

$$
\begin{aligned}
& \hat{\mathcal{R}}=\mathcal{R}_{0}+\sum_{i=1}^{N} \sum_{\mu_{i}} \mathcal{R}_{\mu_{i}} \hat{\tau}_{\mu_{i}}, \\
& \hat{\mathcal{L}}=\mathcal{L}_{0}+\sum_{j=1}^{N} \sum_{v_{j}} \mathcal{L}_{v_{j}} \hat{\tau}_{v_{j}}^{\dagger},
\end{aligned}
$$

where $\mathcal{R}_{\mu_{i}}, \mathcal{L}_{v_{j}}$ are right and left state amplitudes for the excitations $\left\{\mu_{i}, v_{j}\right\}$ of excitation levels $i, j$, respectively, noting that the set $\left\{\mathcal{R}_{0}, \mathcal{L}_{0}\right\}$ refers to the Hartree-Fock state. As a similarity transformation does not change the spectrum of $\hat{H}$, the FCI energy may be obtained from the expression in Eq. (3.1), which involves not the full similarity-transformed Hamiltonian in Eq. (2.7), but rather the CCSD similaritytransformed Hamiltonian in Eq. (2.14). Applying the variational condition on Eq. (3.1) gives the EOM-CC eigenvalue equations expressed in terms of $\hat{H}^{*} \hat{T}$

$$
\begin{aligned}
\left\langle v_{j}\left|\hat{H}^{*} \hat{T}\right| \mathcal{R}\right\rangle & =E_{\mathrm{FCI}} \mathcal{R}_{v_{j}}, \\
\mathcal{L}_{\mu_{i}} E_{\mathrm{FCI}} & =\left\langle\mathcal{L}\left|\hat{H}^{*} \hat{T}\right| \mu_{i}\right\rangle,
\end{aligned}
$$

where $|\mathcal{R}\rangle=\hat{\mathcal{R}}|\mathrm{HF}\rangle$ and $\langle\mathcal{L}|=\langle\mathrm{HF}| \hat{\mathcal{L}}$. In EOM-CCSD theory, the CCSD left and right (ground) eigenstates are given by

$$
\begin{aligned}
& \left\langle{ }^{*} \mathcal{L}\right|=\langle\mathrm{HF}|+\left\langle{ }^{*} \bar{t}_{1}\right|+\left\langle{ }^{*} \bar{t}_{2}\right|, \\
& \left|{ }^{*} \mathcal{R}\right\rangle=|\mathrm{HF}\rangle,
\end{aligned}
$$

where $\left\langle{ }^{*} \mathcal{L}\right|$ is expressed in terms of the CCSD Lagrange multipliers.

RSPT may be applied to the EOM-CC eigenvalue equations in Eqs. (3.3a) and (3.3b) where the left, $\langle\mathcal{L}|$, and right, $|\mathcal{R}\rangle$, states are expanded in orders of the perturbation

$$
\langle\mathcal{L}| \equiv\left\langle\mathcal{L}^{(0)}\right|+\langle\delta \mathcal{L}|=\left\langle\mathcal{L}^{(0)}\right|+\left\langle\delta \mathcal{L}^{(1)}\right|+\left\langle\delta \mathcal{L}^{(2)}\right|+\cdots,
$$

$$
|\mathcal{R}\rangle \equiv\left|\mathcal{R}^{(0)}\right\rangle+|\delta \mathcal{R}\rangle=\left|\mathcal{R}^{(0)}\right\rangle+\left|\delta \mathcal{R}^{(1)}\right\rangle+\left|\delta \mathcal{R}^{(2)}\right\rangle+\cdots,
$$

considering the CCSD left and right eigenstates, $\left\langle{ }^{*} \mathcal{L}\right|$ $=\left\langle\left.\mathrm{HF}\right|^{*} \hat{\mathcal{L}}\right.$ and $\left.\left.\right|^{*} \mathcal{R}\right\rangle={ }^{*} \hat{\mathcal{R}}|\mathrm{HF}\rangle$, as the expansion point for the perturbation series, i.e., $\left\langle\mathcal{L}^{(0)}\right|=\left\langle^{*} \mathcal{L}\right|$ and $\left.\left|\mathcal{R}^{(0)}\right\rangle=\left.\right|^{*} \mathcal{R}\right\rangle$. This is the route taken in the derivation of the $\operatorname{CCSD}(2)$ model $^{15}$ and the models of the $\mathrm{CC}(2) \mathrm{PT}(n)$ hierarchy. ${ }^{16}$ The variational energy in Eq. (3.1) may within this parametrization be expressed as

$$
E_{\mathrm{FCI}}=\frac{\left\langle\mathrm{HF}\left|\left({ }^{*} \hat{\mathcal{L}}+\delta \hat{\mathcal{L}}\right) \hat{H}^{*} \hat{T}\left({ }^{*} \hat{\mathcal{R}}+\delta \hat{\mathcal{R}}\right)\right| \mathrm{HF}\right\rangle}{\left\langle\mathrm{HF}\left|\left({ }^{*} \hat{\mathcal{L}}+\delta \hat{\mathcal{L}}\right)\left({ }^{*} \hat{\mathcal{R}}+\delta \hat{\mathcal{R}}\right)\right| \mathrm{HF}\right\rangle} .
$$

In the Lagrangian approach, perturbation corrections to the CCSD energy are obtained from a variational energy expression of the form

$$
L_{\mathrm{FCI}}=\left(\langle\mathrm{HF}|+\left\langle{ }^{*} \bar{t}|+\langle\delta \bar{t}|) \exp (-\delta \hat{T}) \hat{H}^{*} \hat{T} \exp (\delta \hat{T}) \mid \mathrm{HF}\right\rangle,\right.
$$

where $\delta \hat{T}$ and the set $\{\langle\delta \bar{t}|\}$ contain cluster amplitudes and Lagrange multipliers referencing all excitation levels and the set $\left\{\left\langle{ }^{*} \bar{t}\right|\right\}$ contains the CCSD multipliers of Eq. (3.4a). By parametrizing the exponential operator, $\exp (\delta \hat{T})$, in terms of a linear expansion

$$
\exp (\delta \hat{T})=1+\delta \hat{\mathcal{R}}
$$

we may write Eq. (3.7) as Eq. (3.6) by using Eqs. (3.4a) and (3.4b) and making the identification

$$
\begin{gathered}
\frac{\left.\langle\mathrm{HF}|{ }^{*} \hat{\mathcal{L}}+\delta \hat{\mathcal{L}}\right)}{\left\langle\mathrm{HF}\left|\left({ }^{*} \hat{\mathcal{L}}+\delta \hat{\mathcal{L}}\right)\left({ }^{*} \hat{\mathcal{R}}+\delta \hat{\mathcal{R}}\right)\right| \mathrm{HF}\right\rangle} \\
=\left\langle{ }^{*} \mathcal{L}\left|-{ }^{*} \mathcal{L}\right| \delta \hat{\mathcal{R}}+\langle\delta \bar{t}|-\langle\delta \bar{t}| \delta \hat{\mathcal{R}} .\right.
\end{gathered}
$$

When the CC Lagrangian technique in Eq. (3.7) is used, we have thus parametrized the right state in terms of an exponential ansatz while the left state is parametrized in terms of a linear ansatz. On the contrary, both the right and left states are represented by linear parametrizations in the EOM-CC approach in Eq. (3.6). We note that for first-order wave function corrections, an exponential and a linear parametrization will produce identical results by definition, as the linear terms are the same in the two expansions.

Examining the target energy for the approaches based on Lagrangian and EOM-CC theory reveals how the two approaches exhibit fundamentally different characteristics; while the Lagrangian approach by construction converges towards the target CC energy referenced in the derivation of the series, the target energy for the EOM-CC problem in Eq. (3.6) can only be the FCI energy. Namely, if Eq. (3.7) is truncated at the level of quadruple excitations, we obtain 
$L_{\mathrm{TQ}}$ of Eq. (2.13), for which the target energy is the CCSDTQ energy, cf. Eq. (2.22a). On the other hand, if Eq. (3.6) is truncated at the level of quadruples, neither the CCSDTQ nor the CISDTQ energy is obtained. This is so as the CISDTQ energy is only obtained from Eq. (3.6) if $\hat{H}^{*} \hat{T}$ is replaced by the bare Hamiltonian, $\hat{H}$, while for the CCSDTQ energy to be obtained from Eq. (3.6), the CCSD similarity-transformed Hamiltonian, $\hat{H}^{*} \hat{T}$, has to be replaced by an analogue CCSDTQ similarity-transformed Hamiltonian.

Thus, perturbation theory applied to the EOM-CC problem truncated at any given excitation level will not converge towards the CC energy corresponding to that particular truncated excitation level. It is only in the limit of inclusion of all excitation levels that the target energy becomes well-defined, i.e., the FCI energy, and in this limit Eq. (3.6) is nothing but a reformulation of the FCI problem. In summary, while the $\operatorname{CCSD}(\mathrm{T}-n)$ and $\mathrm{CCSD}(\mathrm{TQ}-n)$ series may be used for examining the convergence from the CCSD towards the CCSDT and CCSDTQ energies-by exploiting their well-defined target energies-such an investigation cannot be performed using any EOM-CC series.

Finally, we note that the linear parametrizations used for describing the left and right states in EOM-CC theory imply that the energy corrections from the EOM-CC problem, truncated at any given excitation level, will suffer from the same size-extensivity problems as are present in truncated CI theory. 35,36

\section{B. Perturbation corrections to the CCSD energy within the EOM-CC picture}

In the context of determining corrections to the CCSD energy using the EOM-CC technique, the HF state and the composite space generated by all singly and doubly excited states from the HF reference is traditionally referred to as the primary CCSD space, while the complementary space, made up from all triply, quadruply, etc., excited states, is referred to as the secondary CCSD space. The primary and secondary spaces may be generated by the projection operators $\hat{P}$ and $\hat{Q}=\hat{I}-\hat{P}$, respectively, where $\hat{I}$ is the identity operator.

When applying RSPT to the EOM-CC problem formulated as in Eqs. (3.1), (3.3a), and (3.3b), the correction to the CCSD energy at $n$ th-order may be obtained from the perturbation series

$$
E^{(n)}=\left\langle\mathcal{L}^{(0)}\left|\hat{H}^{(1)}\right| \delta \mathcal{R}^{(n-1)}\right\rangle,
$$

where the wave function correction to the right state is obtained from the recursion relation ${ }^{16}$

$$
\left(E_{\mathrm{CCSD}}^{(0)}-\hat{H}^{(0)}\right)\left|\delta \mathcal{R}^{(i)}\right\rangle=\hat{H}^{(1)}\left|\delta \mathcal{R}^{(i-1)}\right\rangle-\sum_{j=1}^{i} E^{(j)}\left|\delta \mathcal{R}^{(i-j)}\right\rangle,
$$

where $E_{\mathrm{CCSD}}^{(0)}$ is the CCSD energy. In Eqs. (3.10) and (3.11), $\hat{H}^{(1)}$ refers to the perturbation operator and $\hat{H}^{(0)}$ to the zerothorder Hamiltonian.

Formulating RSPT as in Eqs. (3.10) and (3.11) means that only corrections to the right eigenstate in Eq. (3.5b) are considered, and the energy corrections in Eq. (3.10) will thus satisfy a $n+1$ rule. In order to obtain simplified expressions that satisfy the $2 n+1$ and $2 n+2$ rules, the determination of wave function corrections to both the left and right eigenstates in Eqs. (3.5a) and (3.5b) is required, as was done in the derivation of the $\operatorname{CCSD}(\mathrm{T}-n)$ and $\operatorname{CCSD}(\mathrm{TQ}-n)$ series where the right state parameters (the cluster amplitudes) and the left state parameters (the Lagrange multipliers) obeyed a $2 n+1$ and $2 n+2$ rule, respectively.

As is apparent from Eqs. (3.10) and (3.11), one is faced with the problem of identifying an appropriate zeroth-order Hamiltonian when RSPT is applied within an EOM-CC frame. In principle, this is also true in the case of a derivation of perturbative corrections based on the Lagrangian framework, but here the Møller-Plesset partitioning is an obvious choice. In the context of EOM-CC perturbation theory, the natural choice is less intuitive since one often wants to impose a number of requirements on the zeroth-order Hamiltonian. Commonly, the following two have been imposed: (i) $\hat{H}^{(0)}$ should return the CCSD energy, $E_{\mathrm{CCSD}}^{(0)}$, when evaluated within the primary $\operatorname{CCSD}$ space with $\left\langle{ }^{*} \mathcal{L}\right|$ and $\left|{ }^{*} \mathcal{R}\right\rangle$ as left and right eigenstates, and (ii) the matrix representation of $\left(E_{\mathrm{CCSD}}^{(0)}-\hat{H}^{(0)}\right)$ should be diagonal and contain orbital energy differences (as in ordinary MBPT) within the secondary CCSD space. These requirements are obviously fulfilled if the zeroth- and first-order Hamiltonians, $\hat{H}^{(0)}$ and $\hat{H}^{(1)}$, are defined as in Ref. 16

$$
\begin{gathered}
\hat{H}^{(0)}=\hat{P} \hat{H}^{*} \hat{T} \hat{P}+\hat{Q}\left(E_{\mathrm{CCSD}}^{(0)}+\hat{f}-\sum_{i}^{\text {occ }} \epsilon_{i}\right) \hat{Q}, \\
\hat{H}^{(1)}=\hat{H}^{*} \hat{T}-\hat{H}^{(0)},
\end{gathered}
$$

with $\hat{f}$ being the Fock operator of Eq. (2.9).

In order to construct any size-extensive perturbation series, the zeroth-order Hamiltonian must be additively separable. $\hat{H}^{(0)}$ in Eq. (3.12a), however, does not separate additively due to the presence of projection operators in the $\hat{P} \hat{H}^{*} \hat{T} \hat{P}$ term. ${ }^{38,40}$ Thus, if RSPT is applied to the EOM-CC problem in Eq. (3.6) using the partitioning scheme in Eqs. (3.12a) and (3.12b), the form of $\hat{H}^{(0)}$ will give rise to a perturbation series in which the size extensivity of the energy corrections for a given order is violated, irrespective of whether any restrictions are imposed on the excitation level for the EOM-CC linearly parametrized left and right states.

\section{Comparison of the $\mathrm{CC}(2) \mathrm{PT}(n)$ and $\operatorname{CCSD}(\mathrm{TQ}-n)$ series}

The CC(2)PT $(n)$ series has been derived in accordance with the RSPT energy corrections in Eq. (3.10) and the Hamiltonian partitioning in Eqs. (3.12a) and (3.12b), while the CCSD(TQ- $n$ ) series was derived by means of the Lagrangian technique and a partitioning of the Hamiltonian into the similarity-transformed Fock operator, $\hat{f}^{*} \hat{T}$, and fluctuation potential, $\hat{\Phi}^{*} \hat{T}$. As is readily seen from Eqs. (3.12a) and (3.12b), the matrix representation of the perturbation operator used for deriving the $\mathrm{CC}(2) \mathrm{PT}(n)$ series has no component in the primary space, since $\hat{P} \hat{H}^{(1)} \hat{P}=0$, whereas this is not true in the case of the $\operatorname{CCSD}(\mathrm{TQ}-n)$ series where $\hat{P} \hat{\Phi}^{*} \hat{T} \hat{P} \neq 0$. This implies that the treatment of primary space relaxation in the $\mathrm{CC}(2) \mathrm{PT}(n)$ series is different from that 
in the CCSD(TQ- $n)$ series. This difference between the two schemes, alongside the different parametrizations, cf. Sec. III A, inevitably leads to different paths away from the CCSD energy expansion point in the two series, that is, to different convergence behaviours of the two schemes. We describe below how through third order in the perturbation, the energy corrections of the two series-when $\mathrm{CC}(2) \operatorname{PT}(n)$ is restricted to triple and quadruple excitationsare identical, while the two series differ at fourth and higher order.

Using the Lagrangian technique, energy corrections through third order can be obtained from first-order wave function corrections, when they are derived in accordance with the $2 n+1$ and $2 n+2$ rules. As mentioned previously, first-order corrections obtained using exponential and linear parametrizations will be identical by construction. Furthermore, the first-order wave function correction to the CCSD state contains only components in the triples and quadruples excited manifolds, and for these components the matrix representation of the $\left(E_{\mathrm{CCSD}}^{(0)}-\hat{H}^{(0)}\right)$ operator entering in Eq. (3.11) is given by orbital energy differences (requirement (ii) on $\hat{H}^{(0)}$ in Sec. III B). In the CCSD(TQ- $n$ ) series, the matrix representation of the zeroth-order Hamiltonian (i.e., the similarity-transformed Fock operator) leads to orbital energy differences at all excitation levels, and orders are rigorously counted in the fluctuation potential. Formulated alternatively, the orbital energy differences in the triples and quadruples spaces effectively identify the zeroth-order Hamiltonian in the $\operatorname{CC}(2) \operatorname{PT}(n)$ series and, hence, the fluctuation potential as the perturbation. Thus, as long as the corrections of the $\mathrm{CC}(2) \mathrm{PT}(n)$ series only contain terms that reference the triples and quadruples spaces, this series will effectively count orders in the fluctuation potential and be indistinguishable from the Lagrangian-based perturbation series of the present work.

When the $n+1$ rule is used for evaluating third-order energy corrections, the second-order correction to the wave function, $\left|\delta \mathcal{R}^{(2)}\right\rangle$, is required, which contains also components in the singles and doubles spaces (note that these are different from the ones in $\delta t^{(2)}$ of the Lagrangian approach). However, as $\hat{P} \hat{H}^{(1)} \hat{P}=0$, it may be recognized from Eq. (3.10) that the singles and doubles components of $\left|\delta \mathcal{R}^{(2)}\right\rangle$ effectively vanish in the evaluation of $E^{(3)}$.

While in theory, the secondary space of the CC(2) PT $(n)$ series is unrestricted, a pragmatic truncation at a given excitation level is in practice imposed on the series, cf. the models discussed in Sec. I. For instance, third-order quintuples and hextuples energy corrections are in the $\mathrm{CC}(2) \mathrm{PT}(3)_{\mathrm{TQ}}$ model neglected by restricting the secondary space to the level of quadruple excitations. Imposing this restriction on the $\mathrm{CC}(2) \mathrm{PT}(n)$ hierarchy, only the triples and quadruples spaces are referenced in the energy corrections through third order, and these are thus identical to those of the CCSD(TQ $-n)$ series, which may also be verified by a direct comparison. However, whereas a limitation of the secondary space to the level of quadruple excitations is artificial in the $\mathrm{CC}(2) \mathrm{PT}(n)$ series, it is inherent to the $\operatorname{CCSD}(\mathrm{TQ}-n)$ series, which is the only of the two series that converges towards the CCSDTQ energy.
When fourth-order corrections to the CCSD energy are considered, relaxation effects in the singles and doubles spaces are bound to enter any perturbation series constructed on top of a zeroth-order CCSD problem, since secondorder amplitudes are required. When $\mathrm{CC}(2) \mathrm{PT}(n)$ fourth- and higher-order corrections to the CCSD energy are determined, the $\hat{P} \hat{H}^{*} \hat{T} \hat{P}$ part of $\hat{H}^{(0)}$ will be referenced, and the corrections will per se not be size extensive, as also noted in the Errata $^{41}$ to Ref. 16 where problems related to size extensivity were found at orders $n>3$. In conclusion, the CCSD(TQ $-n)$ and $\mathrm{CC}(2) \mathrm{PT}(n)$ series (even when the $\mathrm{CC}(2) \mathrm{PT}(n)$ series is restricted to contain only triples and quadruples effects) thus differ from each other at fourth and higher orders.

\section{Comparison of biorthogonal MMCC and Lagrangian-based theory}

A different, non-perturbative methodology that too makes usage of the EOM-CC engine, but which takes its stand in approximations to the left eigenstate, $\langle\delta \mathcal{L}|$, while keeping the right eigenstate fixed at $\left|{ }^{*} \mathcal{R}\right\rangle$, has been developed by Piecuch and co-workers. ${ }^{19-21}$ In their approach, approximations are made to $\langle\delta \mathcal{L}|$ based on the knowledge available from the EOM-CCSD eigenvalue equation. This approach is denoted as the MMCC and from it, CR versions of, e.g., the $\operatorname{CCSD}(\mathrm{T})$ and $\operatorname{CCSD}(\mathrm{TQ})$ models ${ }^{19}$ have been derived. In an extension to MMCC theory, the so-called biorthogonal MMCC formalism, ${ }^{20,21}$ corrections to the CCSD energy from triples and quadruples, $\delta_{0}(2,3)_{\mathcal{L}}$ and $\delta_{0}(2,4)_{\mathcal{L}}$, respectively, have been derived. These corrections are similar to the $E_{\mathrm{T}}^{(2)}$ and $E_{\mathrm{Q}}^{(2)}$ corrections of Sec. II C, however, the denominators involved in the expressions for these energy corrections are those of Epstein-Nesbet second-order perturbation theory, i.e., $D_{\mu_{n}}=E_{\mathrm{CCSD}}-\left\langle\mu_{n}\left|\hat{H}^{*} \hat{T}\right| \mu_{n}\right\rangle$ for $n=3$, 4, instead of the orbital energy differences of Møller-Plesset perturbation theory.

\section{SUMMARY AND CONCLUSION}

We have derived a series of perturbative corrections to the CCSD energy that converges towards the CCSDT or CCSDTQ energy, depending on the target energy used in the derivation. The coupled cluster energy Lagrangian technique has been used, considering cluster amplitudes and Lagrange multipliers as variational parameters and parametrizing the target energy around a CCSD reference energy point. In the derivation, the Hamiltonian has been partitioned as in Møller-Plesset perturbation theory, thus considering the fluctuation potential as the perturbation. By virtue of the Lagrangian formulation, the resulting energy corrections satisfy a $2 n+1$ rule for the cluster amplitudes and a $2 n+2$ rule for the multipliers. Explicit expressions have been derived for the energy corrections through fourth order and these are shown to be term-wise size extensive.

Perturbative corrections to the CCSD energy have previously been derived using the EOM-CC formalism. The major difference between the present Lagrangian-based $\operatorname{CCSD}(\mathrm{T}-n)$ and $\operatorname{CCSD}(\mathrm{TQ}-n)$ series and, e.g., the $\mathrm{CC}(2) \mathrm{PT}(n)$ 
series, which is based on EOM-CC theory, lies in the fact that the right eigenstate of the Lagrangian-based series is parametrized in terms of an exponential cluster operator, while in the series based upon EOM-CC theory it is parametrized using linear cluster operators. This implies that the $\mathrm{CC}(2) \mathrm{PT}(n)$ series-as well as any other perturbation series build on top of EOM-CC theory-can only target the FCI energy, whereas the Lagrangian-based series of the present work may target any CC energy, depending only on what target energy is referenced in the derivation of the series. Furthermore, if the secondary space of an EOM-CC perturbation series is truncated, its energy corrections will in general not be size extensive. As an example of the well-defined target energy of a Lagrangian-based series, the convergence behaviour from the CCSD towards the CCSDT energy may be investigated using the $\operatorname{CCSD}(\mathrm{T}-n)$ series, exploiting fully that the series is build on top of the CCSDT coupled cluster Lagrangian.

Different partitioning schemes for separating the total Hamiltonian into a zeroth-order component and a perturbation have also been used in the derivation of the CCSD(T$n) / \mathrm{CCSD}(\mathrm{TQ}-n)$ and $\mathrm{CC}(2) \mathrm{PT}(n)$ series. In the Lagrangianbased series, the similarity-transformed Fock operator acts as the zeroth-order Hamiltonian, and since this operator separates additively, its use leads readily to a size-extensive perturbation series. On the contrary, the zeroth-order Hamiltonian used in the derivation of the $\mathrm{CC}(2) \mathrm{PT}(n)$ series is not additively separable, and the $\mathrm{CC}(2) \mathrm{PT}(n)$ series gives energy corrections that are not size extensive at orders $n>3$. As a result, while the CCSD(TQ- $n$ ) and $\mathrm{CC}(2) \mathrm{PT}(n)$ series are identical through third order-when a pragmatic restriction is imposed on the latter to neglect excitations higher than quadruplesthe two series give different energy corrections at fourth and higher orders.

As the target energies for our derived Lagrangian-based series are well-defined, the fourth- and higher-order corrections from the $\operatorname{CCSD}(\mathrm{T}-n)$ and $\operatorname{CCSD}(\mathrm{TQ}-n)$ series are expected to be useful in difficult cases where relaxation effects in the singles and doubles spaces cannot be neglected, that is, in cases where leading-order corrections from triple and quadruple excitations are not sufficient for an adequate description. Finally, we stress the generality of the present Lagrangian framework, which is readily extended to higher-level parent CC models.

\section{ACKNOWLEDGMENTS}

J.J.E., K.K., T.K., and P.J. acknowledge support from the European Research Council under the European Union's (EU) Seventh Framework Programme (FP/2007-2013)/ERC Grant Agreement No. 291371 and the Danish Council for Independent Research - Natural Sciences. J.G. acknowledges fruitful discussions with Professor Anna I. Krylov (University of
Southern California) and financial support from the Deutsche Forschungsgemeinschaft (DFG GA 370/5-1).

${ }^{1}$ J. Čížek, J. Chem. Phys. 45, 4256 (1966); J. Paldus, J. Čížek, and I. Shavitt, Phys. Rev. A 5, 50 (1972).

${ }^{2}$ G. D. Purvis and R. J. Bartlett, J. Chem. Phys. 76, 1910 (1982).

${ }^{3}$ M. Urban, J. Noga, S. J. Cole, and R. J. Bartlett, J. Chem. Phys. 83, 4041 (1985).

${ }^{4}$ K. Raghavachari, G. W. Trucks, J. A. Pople, and M. Head-Gordon, Chem. Phys. Lett. 157, 479 (1989).

${ }^{5}$ R. J. Bartlett, J. D. Watts, S. A. Kucharski, and J. Noga, Chem. Phys. Lett. 165, 513 (1990).

${ }^{6}$ K. Raghavachari, J. A. Pople, E. S. Replogle, and M. Head-Gordon, J. Phys. Chem. 94, 5579 (1990).

${ }^{7}$ S. A. Kucharski and R. J. Bartlett, Chem. Phys. Lett. 206, 574 (1993).

${ }^{8}$ J. D. Watts, J. Gauss, and R. J. Bartlett, J. Chem. Phys. 98, 8718 (1993).

${ }^{9}$ M. J. O. Deegan and P. J. Knowles, Chem. Phys. Lett. 227, 321 (1994).

${ }^{10}$ S. A. Kucharski and R. J. Bartlett, J. Chem. Phys. 108, 5243 (1998).

${ }^{11}$ S. A. Kucharski and R. J. Bartlett, J. Chem. Phys. 108, 9221 (1998).

${ }^{12}$ T. D. Crawford and J. F. Stanton, Int. J. Quantum Chem. 70, 601 (1998).

${ }^{13}$ Y. J. Bomble, J. F. Stanton, M. Kállay, and J. Gauss, J. Chem. Phys. 123, 054101 (2005)

${ }^{14}$ S. R. Gwaltney and M. Head-Gordon, Chem. Phys. Lett. 323, 21 (2000).

${ }^{15}$ S. R. Gwaltney and M. Head-Gordon, J. Chem. Phys. 115, 2014 (2001).

${ }^{16}$ S. Hirata, M. Nooijen, I. Grabowski, and R. J. Bartlett, J. Chem. Phys. 114, 3919 (2001)

${ }^{17}$ S. Hirata, P.-D. Fan, A. A. Auer, M. Nooijen, and P. Piecuch, J. Chem. Phys. 121, 12197 (2004).

${ }^{18}$ T. Shiozaki, K. Hirao, and S. Hirata, J. Chem. Phys. 126, 244106 (2007).

${ }^{19}$ K. Kowalski and P. Piecuch, J. Chem. Phys. 113, 18 (2000).

${ }^{20} \mathrm{P}$. Piecuch and M. Włoch, J. Chem. Phys. 123, 224105 (2005).

${ }^{21}$ P. Piecuch, M. Włoch, J. R. Gour, and A. Kinal, Chem. Phys. Lett. 418, 467 (2006).

${ }^{22}$ M. Kállay and J. Gauss, J. Chem. Phys. 123, 214105 (2005).

${ }^{23}$ M. Kállay and J. Gauss, J. Chem. Phys. 129, 144101 (2008).

${ }^{24}$ I. Shavitt and R. J. Bartlett, Many-Body Methods in Chemistry and Physics: Many-Body Perturbation Theory and Coupled-Cluster Theory (Cambridge University Press, Cambridge, UK, 2009).

${ }^{25}$ J. Noga and R. J. Bartlett, J. Chem. Phys. 86, 7041 (1987); G. E. Scuseria and H. F. Schaefer, Chem. Phys. Lett. 152, 382 (1988).

${ }^{26}$ N. Oliphant and L. Adamowicz, J. Chem. Phys. 95, 6645 (1991); S. A. Kucharski and R. J. Bartlett, ibid. 97, 4282 (1992).

${ }^{27}$ J. F. Stanton, W. N. Lipscomb, D. H. Magers, and R. J. Bartlett, J. Chem. Phys. 90, 1077 (1989).

${ }^{28}$ T. H. Dunning, Jr., K. A. Peterson, D. E. Woon, and A. K. Wilson, in Proceedings of the American Conference on Theoretical Chemistry, 1999 (unpublished)

${ }^{29}$ A. G. Taube and R. J. Bartlett, J. Chem. Phys. 128, 044110 (2008).

${ }^{30}$ M. Musial and R. J. Bartlett, J. Chem. Phys. 133, 104102 (2010).

${ }^{31}$ A. G. Taube and R. J. Bartlett, J. Chem. Phys. 128, 044111 (2008).

${ }^{32}$ J. F. Stanton and R. J. Bartlett, J. Chem. Phys. 98, 7029 (1993).

${ }^{33}$ J. F. Stanton, Chem. Phys. Lett. 281, 130 (1997).

${ }^{34}$ P. O. Löwdin, J. Math. Phys. 3, 969 (1962).

${ }^{35}$ H. Sekino and R. Bartlett, J. Adv. Quantum Chem. 35, 149 (1999).

${ }^{36}$ M. Nooijen, K. R. Shamasundar, and D. Mukherjee, Mol. Phys. 103, 2277 (2005).

${ }^{37}$ H. Koch, O. Christiansen, P. Jørgensen, A. Sanchez de Merás, and T. Helgaker, J. Chem. Phys. 106, 1808 (1997).

${ }^{38}$ T. Helgaker, P. Jørgensen, and J. Olsen, Molecular Electronic-Structure Theory, 1st ed. (Wiley \& Sons, Ltd., West Sussex, England, 2000).

${ }^{39}$ K. Kristensen, P. Jørgensen, A. J. Thorvaldsen, and T. Helgaker, J. Chem. Phys. 129, 214103 (2008).

${ }^{40}$ J. J. Eriksen, P. Jørgensen, J. Olsen, and J. Gauss, "Equation-of-motion coupled cluster perturbation theory revisited," J. Chem. Phys. (unpublished).

${ }^{41}$ S. Hirata, M. Nooijen, I. Grabowski, and R. J. Bartlett, J. Chem. Phys. 115, 3967 (2001). 